\title{
On the Diffusion in Solids under Finite Deformation
}

\author{
A.G. Varias*
}

Ministry of Defense, Hellenic Navy, Athens, Greece

\begin{abstract}
The governing equations of diffusion in solids, undergoing finite deformation, are presented. Lagrangian and Eulerian descriptions are provided. The governing equations are particularly useful in cases of diffusion-driven degradation mechanisms ahead of cracks in metals, when crack tip blunting is not negligible. Emphasis is placed on hydrogeninduced embrittlement.
\end{abstract}

\section{INTRODUCTION}

A substance, which diffuses in a deforming solid, is considered. The conservation of the diffusing mass is examined in the case of finite strains of the solid. The analysis is general and therefore it can be used in problems of different materials, as is the case of water diffusion in wood or carbon diffusion in iron. Emphasis is placed in metals, where diffusion is involved in most changes of their structure. The accurate mathematical description of diffusion is very important in several degradation mechanisms. Hydrogen induced embrittlement and fracture is a diffusion-driven degradation mechanism, observed in several metals (e.g. iron, nickel, titanium, zirconium) and has important industrial implications. In the case of steady-state hydrogen induced crack growth, the deformation of the metal is adequately described by infinitesimal displacement gradient theory [1]. In this case, the well-known equation of hydrogen mass conservation, which does not distinguishes between the non-deformed and the deformed body, is used. The same equation is also correctly used in the case of diffusion ahead of a stationary crack in an elastically deforming solid [2,3]. All the abovementioned studies take into account the coupling of the operating physical mechanisms of material deformation, hydrogen diffusion and hydride precipitation. In the study by Varias and Massih [2], non-mechanical energy flow is also considered, based on the thermodynamic treatment of coupled phenomena [4]. In the case of a stationary crack in a plastically deforming solid, the blunting of the tip is significant and the consideration of finite deformation near the crack tip is necessary. Indeed in several hydrogen embrittlement studies, finite deformation of the crack tip is simulated [5-7]. Coupling of the operating physical mechanisms is also considered, in order to improve the accuracy of the calculations. However, the effect of finite deformation of metal on hydrogen mass conservation is neglected in all previous studies. In other words, two different theories are applied in these studies, i.e. a finite displacement gradient theory for material deformation and an infinitesimal displacement gradient theory for hydrogen mass conservation. Irrespective of the mathematical inconsistency, the finite deformation effect on hydrogen mass conservation can be important, depending

*Address correspondence to this author at the Makedonias 17, N. Iraklio 141 21, Athens, Greece; E-mail: smro@hol.gr on the velocity of the particles of the metal, where hydrogen lies in interstitial and trap sites or in the form of hydrides. The present discussion provides the finite deformation framework for diffusion and corrects the above-mentioned inconsistency.

In the following, standard tensor notation is used throughout. Bold-faced symbols are used to denote vectors and second-order tensors. Products are indicated with dots and products containing no dots are dyadic products. Latin indices range from one to three and repeated Latin indices are always summed. Inverses, transposes and transposed inverses are denoted with a superscripted $-1, T$ and $-T$, respectively. For example:
$\mathbf{A} \cdot \mathbf{B}=A_{i k} B_{k j} \mathbf{b}_{i} \mathbf{b}_{j}$
$\mathbf{c d}=c_{i} d_{j} \mathbf{b}_{i} \mathbf{b}_{j}$
$\mathbf{c} \cdot \mathbf{d}=c_{i} d_{i}$
$\mathbf{B} \cdot \mathbf{c}=B_{i k} c_{k} \mathbf{b}_{i}$

The base vectors, $\mathbf{b}_{i}$, are Cartesian and independent of time, $t$.

\section{DIFFUSION EQUATIONS}

The body of a solid is undergoing finite deformation, due to the application of external forces. The deformation is described by the deformation gradient $\mathbf{F}(=\partial \mathbf{x} / \partial \mathbf{X})$, where $\mathbf{x}$ is the position of a solid particle in the deformed configuration at time $t$ (spatial coordinates) and $\mathbf{X}$ is its position in a reference (non-deformed) configuration (material coordinates).

The solid contains a diffusing substance. Let $C$ and $\mathbf{J}$ be the concentration and the flux of the diffusing substance, respectively, both defined on the reference configuration. The concentration can be in moles per unit volume or in any other equivalent measure. Mass conservation of the diffusing substance, in a volume $V_{0}$ of the reference configuration, requires that the rate of the amount of the diffusing substance inside this volume is equal to the amount of the diffusing substance flowing through the boundary $S_{0}$ : 
$\frac{d}{d t} \int_{V_{0}} C d V_{0}+\int_{S_{0}} \mathbf{J} \cdot \mathbf{N} d S_{0}=0$

$\mathbf{N}$ is the outward unit vector, normal to the boundary $S_{0}$. Relation (1) is valid for an arbitrary volume and therefore one derives the respective differential equation, by using divergence theorem:

$$
\frac{d C}{d t}+\nabla_{\mathbf{x}} \cdot \mathbf{J}=0
$$

$(d C / d t)=(\partial C / \partial t)_{\mathbf{X}}$, i.e. time derivation is taken at a solid particle in the reference configuration. $\nabla_{\mathbf{X}} \cdot \mathbf{J}=\partial J_{k} / \partial X_{k}$ is the divergence of the flux of the diffusing substance, calculated with respect to material coordinates. Relation (2) is the governing equation of diffusion on the reference configuration and therefore provides the Lagrangian description of diffusion. In the following, the governing equation of diffusion on the deformed configuration is derived.

Let $c$ and $\mathbf{j}$ be the concentration and the flux of the diffusing substance in the deformed configuration. By taking into account the change of a volume and a surface of the solid due to deformation, one shows:

$$
\begin{aligned}
C & =|\mathbf{F}| c \\
\mathbf{J} & =|\mathbf{F}| \mathbf{F}^{-1} \cdot \mathbf{j}
\end{aligned}
$$

$|\mathbf{F}|$ is the determinant of the deformation gradient. The Eulerian description of diffusion is derived by calculating the terms $d C / d t$ and $\nabla_{\mathbf{x}} \cdot \mathbf{J}$, with respect to $c$ and $\mathbf{j}$, and substituting the results into (2). One shows:

$\frac{d C}{d t}=|\mathbf{F}| \frac{d c}{d t}+c|\mathbf{F}| \nabla \cdot \mathbf{v}$

$\nabla_{\mathbf{X}} \cdot \mathbf{J}=|\mathbf{F}| \nabla \cdot \mathbf{j}$

where $\mathbf{v}$ is the velocity of a solid particle. Also $\nabla=\mathbf{b}_{k} \partial / \partial x_{k}$; therefore the divergence and the gradient are taken with respect to spatial coordinates, i.e. $\nabla \cdot \mathbf{j}=\partial j_{k} / \partial x_{k}$ and $\nabla c=\left(\partial c / \partial x_{k}\right) \mathbf{b}_{k}$. On the deformed configuration, the material time derivative satisfies the relation: $d / d t=\partial / \partial t+\mathbf{v} \cdot \nabla$. In the case of the concentration of the diffusing substance $\quad d c / d t=\partial c / \partial t+\mathbf{v} \cdot \nabla c$, where $\partial c / \partial t=(\partial c / \partial t)_{\mathbf{x}}$ is the partial time derivative, related to a point in space and not to a solid particle in the reference configuration. Substitution of (4a) and (4b) into (2) leads to the differential form for the conservation of the diffusing mass on the deformed configuration:

$\frac{d c}{d t}+c \nabla \cdot \mathbf{v}+\nabla \cdot \mathbf{j}=0$

The second term of left hand side of equation (5) is related to the transport of the diffusing substance due to the motion of the solid. Relation (5) was presented by the author in 2001, in a Malmö University report [8]. Independent proofs of (5) are discussed in the Appendix.

Relation (2) or (5) can be used for the solution of problems of hydrogen diffusion in metals ahead of stationary cracks or ahead of growing cracks, during crack growth initiation. In both cases the finite deformation of the metal, due to crack-tip blunting, is not negligible and the use of finite displacement gradient theory is necessary. Therefore the present description of diffusion is essential for dealing with problems of hydrogen embrittlement in metals.

On the deformed configuration, hydrogen flux, $\mathbf{j}^{H}$, depends on the gradient of hydrogen chemical potential in the solid solution, $\mu^{H}$, as well as on the gradient of temperature, $T$, according to the well-known relation [9]:

$\mathbf{j}^{H}=-\frac{D^{H} c^{H}}{R T}\left(\nabla \mu^{H}+\frac{Q^{H}}{T} \nabla T\right)$

where $D^{H}$ and $Q^{H}$ are the diffusion coefficient and the heat of transport of hydrogen in the solid solution, respectively. $c^{H}$ is the concentration of hydrogen in the deformed configuration. Also $R$ is the gas constant. The gradients are taken with respect to spatial coordinates. The chemical potential of hydrogen in solid solution depends on stress [10]; one shows that, under finite deformation of the solid solution, the chemical potential satisfies the following relation:

$\mu^{H}=\mu^{H, 0}-\operatorname{tr}(\sigma) \frac{\bar{V}^{H}}{3}$

or equivalently:

$\mu^{H}=\mu^{H, 0}-|\mathbf{F}|^{-1} \operatorname{tr}\left(\mathbf{F} \cdot \mathbf{S} \cdot \mathbf{F}^{T}\right) \frac{\bar{V}^{H}}{3}$

$\bar{V}^{H}$ is the molal volume of hydrogen in the solid solution; $\mu^{H, 0}$ is the stress-free chemical potential, for the same hydrogen concentration in the reference configuration as that under stress. $\sigma$ is Cauchy stress, defined on the deformed configuration. $\mathbf{S}$ is the second Piola-Kirchhoff stress, defined on the reference configuration. $t r$ denotes the trace of a tensor.

Based on (6), (3a) and (3b), one derives the flux of hydrogen in the reference configuration:

$$
\mathbf{J}^{H}=-\frac{D^{H} C^{H}}{R T}\left(\nabla_{\mathbf{x}} \mu^{H}+\frac{Q^{H}}{T} \nabla_{\mathbf{x}} T\right) \cdot\left(\mathbf{F}^{-1} \cdot \mathbf{F}^{-T}\right)
$$

where $C^{H}$ is the concentration of hydrogen in the reference configuration. Also the gradients are taken with respect to material coordinates.

Relations (2), (7b) and (8), on the reference configuration, or their equivalent, on the deformed configuration, (5), (6) and (7a), describe hydrogen diffusion in a metal under finite deformation, when hydrogen is in solid solution. If hydride precipitation occurs, modification of hydrogen flux is required, e.g. as the one, which was adopted by Varias and 
Massih [2]. However, one should also take into account that hydride volume fraction in the deformed configuration is different from that in the reference configuration.

\section{CONCLUSIONS}

The mass conservation of a substance diffusing in a solid, which undergoes finite deformation, is derived. Both Lagrangian and Eulerian descriptions are discussed. The present development is complementary to the well-known theories on elasto-plastic finite deformation of ductile solids for the simulation of coupled diffusion-deformation problems. It is also directly applicable to the simulation of hydrogeninduced embrittlement in several metals and corrects mathematical inconsistencies of previous studies.

\section{APPENDIX}

In the following, two additional independent proofs of relation (5) are derived.

Consider a control surface, $S$, in the deformed configuration, which bounds a volume $V$ of the solid. The conservation of the mass of the diffusing substance requires that any variation of the amount of the diffusing substance within the solid, instantaneously of volume $V$, is due to the flow of the diffusing substance through the bounding surface, $S$ :

$\frac{d}{d t} \int_{V} c d V+\int_{S} \mathbf{j} \cdot \mathbf{n} d S=0$

$\mathbf{n}$ is the outward unit vector, normal to the control surface. According to Reynolds transport theorem, the rate of increase of the amount of the diffusing substance inside the control surface, $S$, is equal to the rate of increase of the amount of the diffusing substance, possessed by the solid instantaneously inside the volume $V$, minus the outward flux of the substance, carried by solid mass transport through the surface, $S$ :

$\int_{V} \frac{\partial c}{\partial t} d V=\frac{d}{d t} \int_{V} c d V-\int_{S} c \mathbf{v} \cdot \mathbf{n} d S$

Combination of (9) and (10) leads to:

$\int_{V} \frac{\partial c}{\partial t} d V+\int_{S}(\mathbf{j}+c \mathrm{v}) \cdot \mathbf{n} d S=0$

The differential form is derived, by applying the divergence theorem in (11) and by considering that the above relation is valid for an arbitrary volume $V$ :

$$
\frac{\partial c}{\partial t}+\nabla \cdot \mathbf{j}+\nabla \cdot(c \mathbf{v})=0
$$

Taking into account the definition of material time derivative, one derives from (12) an equivalent expression for the conservation of the diffusing mass, which is identical to (5). The above proof was previously presented by the author in a Malmö University Report [8].

Another proof of relation (5) is the following:

$$
\begin{aligned}
& \frac{d}{d t} \int_{V} c d V+\int_{S} \mathbf{j} \cdot \mathbf{n} d S=0 \Leftrightarrow \\
& \frac{d}{d t} \int_{V} \rho\left(\frac{c}{\rho}\right) d V+\int_{V} \nabla \cdot \mathbf{j} d V=0 \Leftrightarrow \\
& \int_{V}\left[\rho \frac{d}{d t}\left(\frac{c}{\rho}\right)+\nabla \cdot \mathbf{j}\right] d V=0
\end{aligned}
$$

$\rho$ is the density of the solid. Relation (13) is valid for an arbitrary volume and therefore:

$\rho \frac{d}{d t}\left(\frac{c}{\rho}\right)+\nabla \cdot \mathbf{j}=0$

Manipulation of (14) and consideration of continuity equation $(d \rho / d t+\rho \nabla \cdot \mathbf{v}=0)$ leads to relation (5).

\section{REFERENCES}

[1] Varias AG, Feng JL. Simulation of hydride-induced steady-state crack growth in metals - Part I: growth near hydrogen chemical equilibrium. Comput Mech 2004; 34: 339-56.

[2] Varias AG, Massih AR. Hydride-induced embrittlement and fracture in metals - Effect of stress and temperature distribution. J Mech Phys Solids 2002; 50: 1469-510.

[3] Lufrano J, Sofronis P, Birnbaum HK. Modeling of hydrogen transport and elastically accommodated hydride formation near a crack tip. J Mech Phys Solids 1996; 44: 179-205.

[4] Denbigh KG. The Thermodynamics of the Steady State. Methuen: London; 1951.

[5] Sofronis P, McMeeking RM. Numerical analysis of hydrogen transport near a blunting crack tip. J Mech Phys Solids 1989; 37 : 317-50.

[6] Lufrano J, Sofronis P, Birnbaum HK. Elastoplastically accommodated hydride formation and embrittlement. J Mech Phys Solids 1998; 46: 1497-520.

[7] Taha A, Sofronis P. A micromechanics approach to the study of hydrogen transport and embrittlement. Eng Fract Mech 2001; 68: 803-37.

[8] Varias AG. Steady-state crack growth due to hydride-induced embrittlement - Mathematical model and numerical implementation. Malmö University: Malmö; 2001

[9] Shewmon PG. Diffusion in Solids. The Minerals, Metals \& Materials Society: Warrendale, Pennsylvania; 1989.

[10] Li JCM, Oriani RA, Darken LS. The thermodynamics of stressed solids. Z Phys Chem N F 1966; 49: 271-90. 